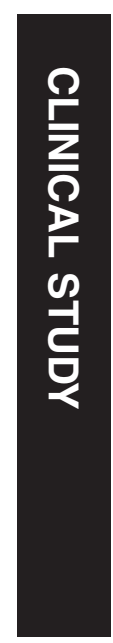

\section{Matched optical quality comparison of 3-year results of PRK-MMC and phakic IOL implantation in the correction of high myopia}

M Miraftab ${ }^{1}$, H Hashemi ${ }^{1}$ and S Asgari²
${ }^{1}$ Noor Ophthalmology Research Center, Noor Eye Hospital, Tehran, Iran

${ }^{2}$ Department of Epidemiology and Biostatistics, School of Public Health, Tehran University of Medical Sciences, International Campus (TUMS-IC),

Tehran, Iran

Correspondence: H Hashemi, Noor Ophthalmology Research Center, Noor Eye Hospital, No. 96 Esfandiar Blvd., Vali'asr Ave, Tehran 1968653111, Iran

Tel: +98 2188651515 ;

Fax: +98 21886514

E-mail: hhashemi@norc.ac.ir

Received: 16 September 2014

Accepted in revised form: 27 March 2015

Published online:

15 May 2015

\begin{abstract}
Aims To compare 3-year results of PRK-MMC and phakic intraocular lens (PIOL) implantation in patients with $>\mathbf{8 . 0}$ diopters (D) of myopia.

Methods This study was conducted as a non-randomized clinical trial on 23 eyes treated with PIOL (Artiflex; group A) and 23 eyes treated with PRK-MMC (group B). This report compares 3-year treatment results in these two groups.

Results At 3 years after surgery, uncorrected visual acuity was $0.02 \pm 0.06$ LogMAR in group $A$ and $0.04 \pm 0.07$ LogMAR in group $B(P=0.639)$. Mean best corrected visual acuity in group $A$ $(0.004 \pm 0.02)$ was better than group $B$ $(0.03 \pm 0.07$ LogMAR $)(P=0.035)$. Mean manifest refraction spherical equivalent was $-0.16 \pm 0.21$ and $-0.09 \pm 0.20 \mathrm{D}(P=0.190)$, respectively. Mesopic contrast sensitivity (CS) in the spatial frequency of three cycle/degree (CS3) significantly decreased in both groups, but the reduction was significantly higher in group $B(P=0.024)$. CS6 decreased significantly only in group $B$ $(P=0.019)$. Changes in CS12 and CS18 showed no significant inter-group difference. In group $\mathrm{A}$, the increase in $\mathrm{C} 6$ trefoil $(0.16 \pm 0.18 \mu \mathrm{m}, P=0.003)$ and reduction in spherical aberration (SA; $0.16 \pm 0.08 \mu \mathrm{m}$, $P<0.001)$ were statistically significant. In group $B$, the reduction in vertical coma $(P=0.052)$, and increases in horizontal coma $(P=0.044)$, coma $(P<0.001)$, SA $(P<0.001)$, and total higher order aberrations $(P<0.001)$ were significant after surgery. Conclusion Based on 3-year results, PIOL implantation is a better choice than
\end{abstract} PRK-MMC for treating patients with $>8.0 \mathrm{D}$ myopia. However, for patients with an inadequate aqueous depth, PRK-MMC can be an acceptable treatment option with a potential for decreased quality of vision. Eye (2015) 29, 926-931; doi:10.1038/eye.2015.71; published online 15 May 2015

\section{Introduction}

Treatment options for the correction of high myopia include refractive surgeries such as photorefractive keratectomy (PRK), laser in situ keratomileusis (LASIK), and intraocular refractive procedures. Clinicians have different opinions on the applications of these methods. Some prefer PRK because of the risk of postLASIK ectasia, and some prefer phakic intraocular lens (PIOL) implantation due to the risk of corneal haze after PRK. ${ }^{1-3}$ We designed a matched comparative study of patients with $>8.0$ diopters (D) of myopia and compared results of treatment with PRK and PIOL in these two groups. Our 1-year results ${ }^{4}$ indicated that surface ablation and PIOL implantation correct uncorrected visual acuity (UCVA), best corrected visual acuity (BCVA), refraction, and astigmatism similarly, and they do not impact mesopic contrast sensitivity (CS). Almost all higher order aberrations (HOAs) increased after PRK-MMC, but PIOL implantation was only associated with increased C6 (trefoil) and total HOA, whereas spherical aberration (SA) decreased.

As assessment of treatment results requires longer follow-up times, here we present 3-year results with these two methods, to report visual function changes in this period. 


\section{Materials and methods}

Study methods have previously been described. ${ }^{4}$ In this non-randomized clinical trial, patients with high myopia (spherical equivalent $\geq 8.0 \mathrm{D}$ and astigmatism $\leq 2.0 \mathrm{D}$ ) who had a stable refraction over the past 12 months were included. Patients with an aqueous depth (AQD) $>3.0 \mathrm{~mm}$ from the epithelium to the lens, as measured by Pentacam HR (Oculus, Wetzlar, Germany), endothelial cell count $>2500 \mathrm{cell} / \mathrm{mm}^{2}$, and insufficient corneal thickness were selected for PIOL implantation. Patients with an AQD $<3.0 \mathrm{~mm}$ or those who were unwilling to have intraocular surgery were treated with PRK-MMC if their postoperative residual stromal bed thickness would be $>350$ microns. Exclusion criteria of the study were having an ocular pathology or a history of ocular surgery. Patients who used contact lenses were instructed to stop wearing them for 4 weeks before surgery. Twenty-three eyes were enrolled in each study group.

After explaining the study objectives and methods, an informed consent was obtained from all patients. The study was approved by the Institutional Review Board, and it adhered to the tenets of the Declaration of Helsinki Principles at all stages.

\section{Surgical techniques}

PRK-MMC With topical anesthesia, the corneal epithelium was scraped off mechanically without alcohol usage. The VISX STAR S4 excimer laser (Abbott, Abbott Park, IL, USA), software version: 5.30 , set for a $6.0 \mathrm{~mm}$ ablation zone and a $1.25 \mathrm{~mm}$ blend zone was used for corneal reshaping. After that, a sponge soaked in mitomycin-C $0.02 \%$ was applied to the ablated stroma for $10 \mathrm{~s}$ per diopter of correction. After irrigation with $30 \mathrm{cc}$ sterile balanced salt solution, a bandage contact lens (Air Optix, Ciba Vision, Duluth, GA, USA) was placed on the cornea. Postoperative eye drops included betamethasone $(0.1 \%)$ four times daily, levofloxacin $(5 \mathrm{mg} / \mathrm{ml})$ four times daily, and artificial tears (Hypromellose, preservative free). Examinations were done daily until complete epithelial healing was observed. Upon re-epithelialization, the contact lens was removed, and levofloxacin was discontinued, but betamethasone and artificial tears were continued for another 2 weeks. After 2 weeks, fluorometholone $(0.1 \%)$ was prescribed and tapered over a course of 3 months.

PIOL implantation In this procedure, topical anesthesia was induced along with IV sedation. A $3.2 \times 1.5 \mathrm{~mm}$ corneal incision was created at 12 o'clock by an appropriate keratome. Then, a spherical Artiflex (Ophtec, Boca Raton, FL, USA) PIOL, which was selected using the software version 7.1 in IOLMaster 500 (Carl Zeiss Meditec AG, Jena,
Germany), was enclavated in the eye horizontally. Peripheral iridotomy was done, and as the incision provided watertight closure, no suturing was done. Postoperative eye drops included levofloxacin for 5 days and betamethasone four times daily for 1 week. Then, fluorometholone (0.1\%) was started and tapered over 2 months.

Patients were examined before surgery, and at 1 month, 6 months, 1 year, and 3 years after surgery. UCVA and BCVA were tested using the Snellen chart, and manifest refraction spherical equivalent (MRSE) was determined with a retinoscope (HEINE BETA 200, Herrsching, Germany). CS was assessed with best distance correction using CVS-1000 grating charts (VectorVision, Inc., Greenville, $\mathrm{OH}$, USA) under mesopic conditions without dilation. The Allegretto WaveLight analyzer (WaveLight Laser Technologie AG, Erlangen, Germany) was used for aberrometry, and $6.0 \mathrm{~mm}$ data were extracted. For each patient, the best of three measurements was used for analysis. Extracted aberrometry parameters were C6 (trefoil), C7 (vertical coma), C8 (horizontal coma), RMS total coma, C9 (trefoil), C12 (SA), and total HOA RMS. Total coma was calculated from the root of the sum of C7 and C8 squares.

In the first report, ${ }^{4}$ the trends of changes over 1 year were compared between the two types of treatment. Here, we compare the two methods in terms of 3-year changes against preoperative. A significance level of 0.05 was chosen.

\section{Results}

Mean preoperative myopia was $-9.49 \pm 1.94 \mathrm{D}(-12.50$ to $-8.00)$ and $-8.82 \pm 1.25 \mathrm{D}$ ( -11.50 to -8.00$)$ in the PIOL and PRK-MMC groups, respectively $(P=0.871)$. Mean preoperative cylindrical error was $-1.27 \pm 0.66 \mathrm{D}(-2.00$ to $0.00)$ and $-0.99 \pm 0.53 \mathrm{D}(-2.00$ to 0.00$)$ in the PIOL and PRK-MMC groups, respectively $(P=0.122)$. Mean age was $27.74 \pm 5.28$ years in the PIOL group and $28.78 \pm 5.34$ years in the PRK-MMC group $(P=0.509)$, and females comprised $73 . \%$ and $60.9 \%$ of the patients in the former and latter groups, respectively $(P=0.345)$. No complications were observed in either group up to 3 years after surgery.

At 3 years after surgery, mean UCVA was $0.02 \pm 0.06$ LogMAR in the PIOL group and $0.04 \pm 0.07$ LogMAR in the PRK-MMC group $(P=0.639)$, and mean BCVA was $(0.004 \pm 0.02 \log M A R$ and $0.03 \pm 0.07 \log M A R$, respectively $(P=0.035)$. In the two groups treated with PIOL and PRK-MMC, mean spherical error was $0.00 \pm 0.00 \mathrm{D}$ and $-0.03 \pm 0.18 \mathrm{D}(P=0.217)$, mean astigmatism was $-0.32 \pm 0.43 \mathrm{D}$ and $-0.11 \pm 0.21 \mathrm{D}$ $(P=0.720)$, and mean MRSE was $-0.16 \pm 0.21 \mathrm{D}$ and $-0.09 \pm 0.20 \mathrm{D}(P=0.190)$, respectively. At the end of the third year, $95.2 \%$ in the PIOL group os $95.8 \%$ in the 
PRK-MMC group were within $\pm 0.50 \mathrm{D}$ of emmetropia $(P=1.000)$ (Table 1).

Mesopic CS changes are summarized in Figure 1. After 3 years, CS3 had significantly decreased in both groups, but the decrease was significantly greater in the PRKMMC group $(P=0.024)$. CS6 showed an increase in the PIOL group and a decrease in the PRK-MMC group; the inter-group difference in C6 change was statistically

Table 1 Three-year results of visual acuity and refraction after phakic IOL (PIOL) implantation and PRK-MMC for myopia $\geq 8.0 \mathrm{D}$

\begin{tabular}{|c|c|c|c|c|c|}
\hline & \multirow[t]{2}{*}{ Before surgery } & \multicolumn{2}{|c|}{ After surgery } & \multirow[t]{2}{*}{ P-value ${ }^{\mathrm{a}}$} & \multirow[t]{2}{*}{ P-value } \\
\hline & & 1 Year & 3 Years & & \\
\hline \multicolumn{6}{|c|}{ UCVA (logMAR) } \\
\hline PIOL & $1.83 \pm 0.24$ & $0.03 \pm 0.07$ & $0.02 \pm 0.06$ & $<0.001$ & 0.639 \\
\hline PRK+MMC & $1.84 \pm 0.29$ & $0.05 \pm 0.08$ & $0.04 \pm 0.07$ & $<0.001$ & \\
\hline \multicolumn{6}{|c|}{$B C V A(\log M A R)$} \\
\hline PIOL & $0.04 \pm 0.07$ & $0.01 \pm 0.02$ & $0.004 \pm 0.02$ & 0.035 & 0.032 \\
\hline $\mathrm{PRK}+\mathrm{MMC}$ & $0.04 \pm 0.07$ & $0.03 \pm 0.07$ & $0.03 \pm 0.07$ & 0.664 & \\
\hline \multicolumn{6}{|l|}{ Sphere (diopter) } \\
\hline PIOL & $-8.86 \pm 1.90$ & $0.02 \pm 0.10$ & $0.00 \pm 0.00$ & $<0.001$ & 0.217 \\
\hline PRK+MMC & $-8.33 \pm 1.35$ & $-0.12 \pm 0.40$ & $-0.03 \pm 0.18$ & $<0.001$ & \\
\hline \multicolumn{6}{|c|}{ Cylinder (diopter) } \\
\hline PIOL & $-1.27 \pm 0.66$ & $-0.39 \pm 0.33$ & $-0.32 \pm 0.43$ & $<0.001$ & 0.720 \\
\hline $\mathrm{PRK}+\mathrm{MMC}$ & $-0.99 \pm 0.53$ & $-0.24 \pm 0.31$ & $-0.11 \pm 0.21$ & $<0.001$ & \\
\hline \multicolumn{6}{|l|}{ MRSE (diopter) } \\
\hline PIOL & $-9.49 \pm 1.94$ & $-0.17 \pm 0.17$ & $-0.16 \pm 0.21$ & $<0.001$ & 0.190 \\
\hline PRK+MMC & $-8.82 \pm 1.25$ & $-0.24 \pm 0.39$ & $-0.09 \pm 0.20$ & $<0.001$ & \\
\hline
\end{tabular}

Abbreviations: BCVA, best corrected visual acuity; MRSE, manifest refraction spherical equivalent; UCVA, uncorrected visual acuity. ${ }^{\text {a Three year results }}$ compared with before surgery. ${ }^{\mathrm{b}}$ Inter-group difference in 3-year changes.

a

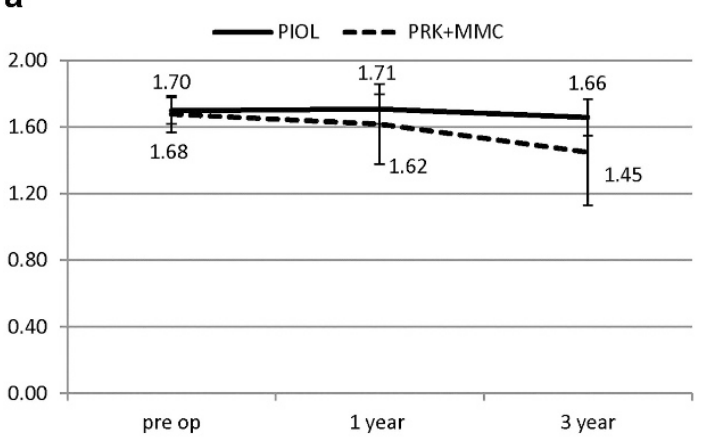

C

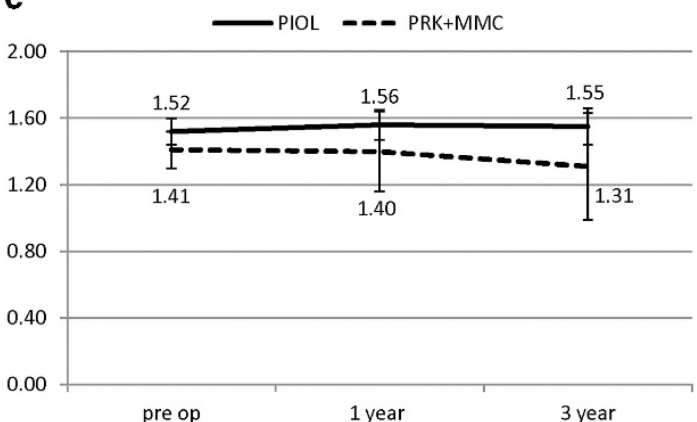

b

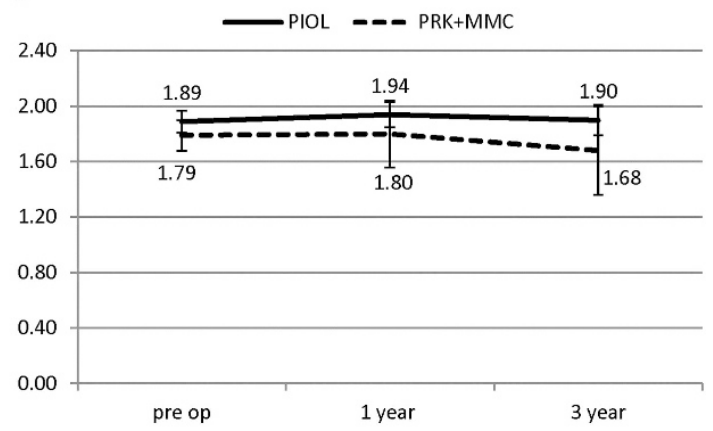

d

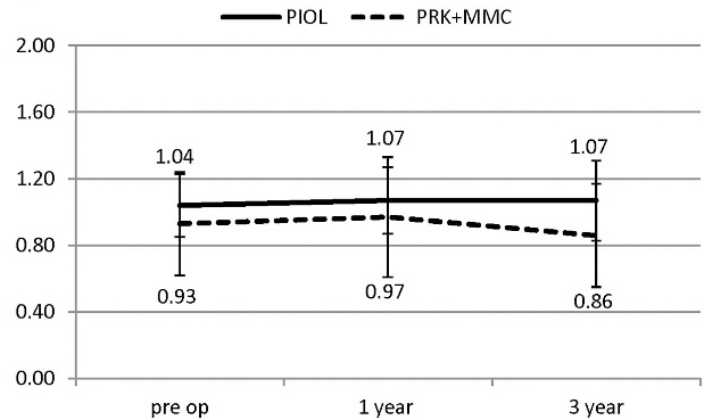

Figure 1 Three-year logarithm of mesopic contrast sensitivity after phakic IOL (PIOL) implantation and PRK-MMC for myopia $\geq 8.0$ D; (a) CS3, (b) CS6, (c) CS12, and (d) CS18. 
a

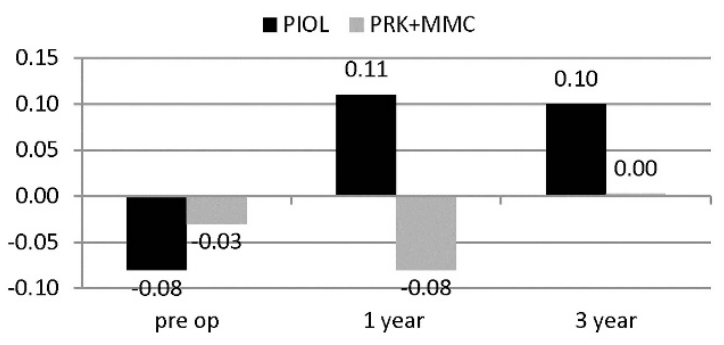

C

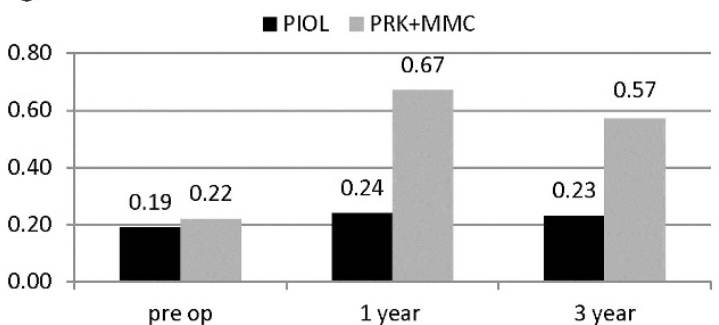

b

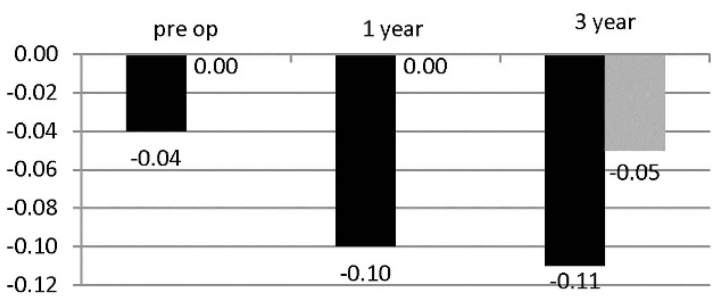

d

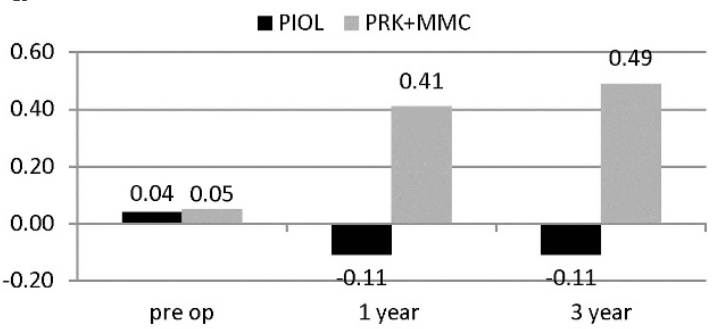

e

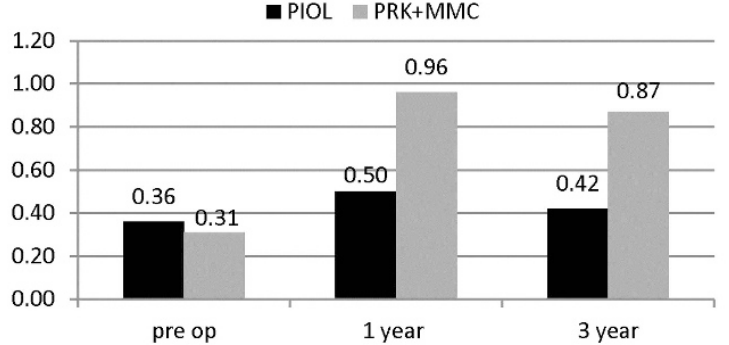

Figure 2 Three-year results of higher order aberrations after phakic IOL (PIOL) implantation and PRK-MMC for myopia $\geq 8$ D; (a) C6 trefoil, (b) C9 trefoil, (c) total coma, (d) spherical aberrations, and (e) total higher order aberrations.

significant $(P=0.039)$. Changes in CS12 and CS18 were not statistically significantly different between the two groups.

Of the aberrations, only the increase in trefoil C6 $(P=0.003)$ and decrease in SA $(P<0.001)$ was statistically significant in the PIOL group. In the PRK-MMC group, the decrease in vertical coma $(P=0.052)$, and increases in horizontal coma $(P=0.044)$, total coma $(P<0.001)$, SA $(P<0.001)$, and total HOA $(P<0.001)$ were significant at 3 years after surgery. Inter-group differences in aberration changes were significant for vertical coma $(P=0.058)$, total coma $(P<0.001)$, SA $(P<0.001)$, and total HOA $(P<0.001$; Figure 2).

\section{Discussion}

In this study, we assessed long-term results of aspheric PIOL (Artiflex) implantation, which is an iris fixated IOL, compared to those with PRK-MMC in the treatment of cases with $>8.0 \mathrm{D}$ of myopia.

At the end of 3 years, $100 \%$ in the PIOL group had BCVA of $20 / 20$ or better. This is while in the PRK-MMC group, $75 \%$ of cases had 20/20 BCVA; the decrease had occurred by the sixth postoperative month and BCVA remained unchanged until the end of the third year. Two eyes in this group lost one line of BCVA. In both these eyes, the preoperative SE was very high $(-12.12 \mathrm{D}$ and $-12.50 \mathrm{D})$. As we know, laser refractive surgery can reduce BCVA at such high myopic corrections, nonetheless, it was only about one Snellen line. As there was no BCVA loss in the PIOL group, we could say PIOL implantation showed better results than PRK-MMC as far as safety is concerned.

Our results with PIOL implantation were similar to those in the study by Silva et $a l^{5}$ but or results with PRKMMC were better than those of similar studies, ${ }^{6,7}$ which can be due to technical improvements in excimer laser machines in this decade. Another possibility is that MMC application improves results, but this hypothesis, at least in myopia $<8.0 \mathrm{D}$, is doubted today. ${ }^{8}$ As BCVA showed no change in the PRK-MMC group from year 1 to the third year after surgery and remained stable, but improved gradually in the PIOL group, we could assume that PIOL implantation offers better long-term safety than 
PRK-MMC in the treatment of high myopia. Had we assessed endothelial cell counts, we would be able to make a stronger judgment.

In terms of predictability, the two methods showed a similar trend over 3 years. No case of SE regression was observed in either group. In the PRK-MMC group, however, two eyes demonstrated 0.25D and 0.5D increase in refractive astigmatism.

In terms of mesopic CS, results with PIOL were better than PRK-MMC at lower spatial frequencies (CS3 and CS6). This difference decreased at higher spatial frequencies to the extent that results were similar in both groups. At spatial frequency of three cycles per degree (CPD), both methods had resulted in CS reduction, but the reduction was less with PIOL. At spatial frequency of six CPD, PIOL implantation showed no change but PRKMMC caused a reduction in CS. At higher frequencies (CS12 and CS18), we found no significant changes in either group, and the two groups were similar in this regard. Our findings in the PIOL group are similar to the study by Lombardo et $a l^{9}$ in which mesopic CS only showed a significant decrease at a spatial frequency of three CPD. This decrease can be due to the increased pupil size under mesopic conditions, and light scatter at the lens edges which increases aberrations and decreases CS. This situation worsened in the presence of IOL decentration.

As we know, the spatial frequency of one CPD is mathematically equivalent to $20 / 600$ on the Snellen chart and 30 CPD is equal to $20 / 20{ }^{10}$ Thus, we can say that higher spatial frequencies are indicators of visual acuity whereas lower spatial frequencies show visual quality. With PRK, aberrations increase because the corneal shape is changed from prolate to oblate, ${ }^{11,12}$ and aberrations increase even more under mesopic conditions, when pupil size increase. ${ }^{13,14}$ Therefore, PRK is expected to have more deteriorating effect than PIOL implantation in spatial frequencies up to CS6, and our results is in concordance with this explanation. The observed increases in SA and total HOA in this group were also in agreement with this hypothesis. The reason we found no inter-group differences at higher frequencies is probably that these CS frequencies are affected more by haze and defocus blur. ${ }^{15}$ As haze and defocus blur resolved, no significant change was seen at higher frequencies.

As discussed, aberrations are expected to increase after refractive surface ablation procedures. The decrease in vertical coma in the PRK-MMC group could be due to the correction of astigmatism. Comparison of 1-year and 3year results in this group showed a decreasing trend for total coma and total HOAs, though higher compared with their preoperative states. Similarly, in the PIOL group, total HOA was increased significantly at 1 year after surgery, but decreased thereafter and returned to the preoperative status.

In conclusion, based on 3-year results, PIOL implantation is preferable to PRK-MMC for refractive surgery in cases with $>8.0 \mathrm{D}$ of myopia. But for cases with inadequate AQD for PIOL implantation, PRK-MMC can be used for refractive correction with the understanding that quality of vision decreases under both photopic and mesopic conditions. However, short-term and long-term complications of intraocular surgical procedures vs PRKMMC and the risk-to-benefit balance should be considered. Regarding rare complications such as glaucoma and cataract, we need longer follow-ups. One of the study limitations was the inability to perform contralateral comparisons as a consequence of the inclusion criteria. Also, the groups had limited sample sizes, although we minimized this limitation by matching preoperative data in the two groups.

\section{Summary}

What was known before

- After 1 year, phakic IOL Implantation was better compared with PRK-MMC in the correction of high myopia in terms of visual quality but two methods had no difference in regards to visual acuity.

What this study adds

- After 3 years, PIOL implantation is a better choice compared with PRK-MMC for treating patients with $>8.0$ diopters myopia. However, for patients with an inadequate aqueous depth, PRK-MMC can be an acceptable treatment option.

\section{Conflict of interest}

The authors declare no conflict of interest.

\section{Acknowledgements}

This study was funded by Noor Eye Hospital.

\section{References}

1 Duffey RJ, Leaming D. US trends in refractive surgery: 2004 ISRS/AAO Survey. J Refract Surg 2005; 21: 742-748.

2 Sia RK, Ryan DS, Edwards JD, Stutzman RD, Bower KS. The U.S. Army Surface Ablation Study: comparison of PRK, MMC-PRK, and LASEK in moderate to high myopia. J Refract Surg 2014; 30: 256-264.

3 Huang D, Schallhorn SC, Sugar A, Farjo AA, Majmudar PA, Trattler WB. Phakic intraocular lens implantation for the correction of myopia: a report by the American Academy of Ophthalmology. Ophthalmology 2009; 116: 2244-2258.

4 Hashemi H, Miraftab M, Asgari S. Comparison of the visual outcomes between PRK-MMC and phakic IOL implantation in high myopic patients. Eye (Lond) 2014; 28: 1113-1118. 
5 Silva RA, Jain A, Manche EE. Prospective long-term evaluation of the efficacy, safety, and stability of the phakic intraocular lens for high myopia. Arch Ophthalmol 2008; 126: 775-781.

6 Alio JL, Ortiz D, Muftuoglu O, Garcia MJ. Ten years after photorefractive keratectomy (PRK) and laser in situ keratomileusis (LASIK) for moderate to high myopia (control-matched study). Br J Ophthalmol 2009; 93: 1313-1318.

7 Rosman M, Alio JL, Ortiz D, Perez-Santonja JJ. Comparison of LASIK and photorefractive keratectomy for myopia from -10.00 to -18.00 diopters 10 years after surgery. J Refract Surg 2010; 26: 168-176.

8 Hofmeister EM, Bishop FM, Kaupp SE, Schallhorn SC. Randomized dose-response analysis of mitomycin- $C$ to prevent haze after photorefractive keratectomy for high myopia. J Cataract Refract Surg 2013; 39: 1358-1365.

9 Lombardo AJ, Hardten DR, McCulloch AG, Demarchi JL, Davis EA, Lindstrom RL. Changes in contrast sensitivity after Artisan lens implantation for high myopia. Ophthalmology 2005; 112: 278-285.
10 Watson TA (ed). General methodology. In: Quantitative Assessment of Vision Function in Children with Cortical Visual Impairment. ProQuest: SC, USA, 2008, pp 27-39.

11 Serrao S, Lombardo G, Ducoli P, Lombardo M. Long-term corneal wavefront aberration variations after photorefractive keratectomy for myopia and myopic astigmatism. I Cataract Refract Surg 2011; 37: 1655-1666.

12 Serrao S, Lombardo G, Ducoli P, Lombardo M. Optical performance of the cornea six years following photorefractive keratectomy for myopia. Invest Ophthalmol Vis Sci 2011; 52: 846-857.

13 O'Brart DP, Lohmann CP, Fitzke FW, Klonos G, Corbett MC, Kerr-Muir MG et al. Disturbances in night vision after excimer laser photorefractive keratectomy. Eye (Lond) 1994; 8: 46-51.

14 O'Brart DP, Lohmann CP, Fitzke FW, Smith SE, Kerr-Muir MG, Marshall J. Night vision after excimer laser photorefractive keratectomy: haze and halos. Eur J Ophthalmol 1994; 4: 43-51.

15 Montes-Mico R, Charman WN. Choice of spatial frequency for contrast sensitivity evaluation after corneal refractive surgery. J Refract Surg 2001; 17: 646-651. 